\title{
ShRNA-mediated Bmi-1 silencing sensitizes multiple myeloma cells to bortezomib
}

\author{
SHUN-QUAN WU*, ZHEN-ZHEN XU*, WEN-YAN NIU, HAO-BO HUANG and RONG ZHAN \\ Fujian Institute of Hematology, Affiliated Union Hospital of Fujian Medical University, \\ Fujian Provincial Key Laboratory on Hematology, Fuzhou, Fujian 350001, P.R. China
}

Received January 13, 2014; Accepted April 29, 2014

DOI: $10.3892 /$ ijmm.2014.1798

\begin{abstract}
The introduction of bortezomib has resulted in a paradigm shift in the treatment of multiple myeloma (MM) and has contributed to the improved survival of patients with MM. Inevitably, resistance to therapy develops, and thus the clinical efficacy of bortezomib is hampered by drug resistance. The oncogene B-cell-specific Moloney murine leukemia virus insertion site-1 (Bmi-1) has been implicated in the pathogenesis of various human malignancies. Furthermore, RNA interference (RNAi)-mediated Bmi-1 silencing has been shown to sensitize tumor cells to chemotherapy and radiation. The role of Bmi-1 in influencing the response to bortezomib therapy has not been investigated to date. In the present study, Bmi-1 was silenced in two MM cell lines (U266 and RPMI8226) using short hairpin RNA (shRNA) targeting Bmi-1 (shBmi-1). A cell counting kit-8 (CCK-8) assay was performed to analyze cell proliferation and evaluate the $50 \%$ inhibitory concentration (IC50) values of bortezomib. Cell cycle progression and apoptosis were analyzed by flow cytometry (FCM), and the mRNA and protein expression of associated genes (Bmi-1, p14, p21, Bcl-2 and Bax) was quantified by RT-qPCR and western blot analysis, respectively. The IC50 values significantly decreased in the cells transfected with shBmi-1 $(\mathrm{p}<0.05)$. The depletion of Bmi-1 sensitized the MM cells to bortezomib, which increased the $G_{1}$ phase duration and enhanced bortezomib-induced apoptosis $(\mathrm{p}<0.05)$. The expression of p21 and Bax (apoptosis inducer) was upregulated whereas that of the anti-apoptotic protein, Bcl-2, was downregulated in the Bmi-1-silenced cells $(\mathrm{p}<0.05)$. The depletion of Bmi-1 enhanced the sensitivity of MM cells to bortezomib by inhibiting cell proliferation and inducing cell cycle arrest and apoptosis.
\end{abstract}

Correspondence to: Professor Rong Zhan, Fujian Institute of Hematology, Affiliated Union Hospital of Fujian Medical University, Fujian Provincial Key Laboratory on Hematology, 29 Xin Quan Road, Fuzhou, Fujian 350001, P.R. China

E-mail: deanzhanrong@qq.com

*Contributed equally

Key words: B-cell-specific Moloney murine leukemia virus insertion site-1, bortezomib, chemosensitivity, multiple myeloma cell, RNA interference
Our data suggest that Bmi-1 may serve as an important novel therapeutic target in MM.

\section{Introduction}

Multiple myeloma (MM) is a type of cancer that arises from the neoplastic proliferation of plasma cells and is characterized by the proliferation of malignant plasma cells into the bone marrow (BM) and the excessive secretion of monoclonal immunoglobulin or Bence Jones proteins. The major clinical manifestations include extensive bone destruction, anemia, hypercalcemia, hyperviscosity and renal dysfunction (1). Of note, the incidence of MM in Western countries is higher than that in Asian countries (2). Despite advances in the understanding of the molecular pathogenesis of $\mathrm{MM}$ and promising new therapies, only $25-35 \%$ of patients respond to therapy in the relapsed and refractory setting $(3,4)$.

The boronic dipeptide, bortezomib, a reversible proteasome inhibitor, has shown marked anticancer activity in various cancer cell types, including MM cells, which are resistant to conventional therapies $(5,6)$. Despite the promising clinical activity, the efficacy of bortezomib may differ among tumor types, and some patients with MM fail to respond to bortezomib therapy (7). Moreover, almost one-third of patients with MM never respond to treatment with bortezomib, depending on the clinical situation (7). Whether these observations are related to the common mechanisms of drug resistance frequently observed for anticancer drugs remains unclear. However, the understanding of the characteristics of drug resistance and the enhancement of the sensitivity to bortezomib may help to overcome drug resistance. In addition, recent evidence has demonstrated that silencing the expression of oncogenes, such as myeloid cell leukemia sequence-1 (Mcl-1) and melanoma antigen gene (MAGE)-C1/CT7, can sensitize MM cells to bortezomib $(8,9)$, suggesting that RNA interference (RNAi) may be an effective method for enhancing the sensitivity of MM cells to bortezomib or even reversing resistance.

B-cell-specific Moloney murine leukemia virus insertion site-1 (Bmi-1), a member of the polycomb family, was initially identified as an oncogene that cooperates with c-Myc in the initiation of lymphoma in murine models $(10,11)$. Over the years, Bmi-1 has been reported to be involved in axial patterning (12), hematopoiesis (13), the regulation of proliferation and senescence (14). It has also been found to be essential for the 
self-renewal of normal and malignant stem cells (15). Several lines of evidence have also indicated that Bmi-1 is extensively upregulated in a variety of malignancies, including non-small cell lung cancer (16), leukemia (17), as well as breast (18), colorectal (19), pancreatic (20) and prostate cancer (21). Moreover, it has been demonstrated that $\mathrm{Bmi}-1$ is overexpressed in $\mathrm{MM}$ and can regulate the growth and clonogenic capacity of MM cells both in vitro and in vivo (22). However, whether Bmi-1 can affect the sensitivity of MM cells to bortezomib is largely unknown. Furthermore, it has been reported that the downregulation of Bmi-1 can result in cancer cell apoptosis and that the RNAi-mediated depletion of Bmi-1 can sensitize tumor cells to chemotherapy and radiotherapy (23-25). Thus, we hypothesized that the abrogation of Bmi-1 expression may be an effective strategy for sensitizing MM cells to bortezomib.

In this study, we demonstrate that Bmi-1 plays an important role in the sensitization of MM cells to bortezomib. To determine the combined effect of RNAi and bortezomib treatment on MM cells in vitro, we introduced a lentiviral interference vector expressing short hairpin RNA (shRNA) to silence Bmi-1 in MM cells. Our results demonstrate that Bmi-1 silencing sensitizes MM cells to bortezomib by inhibiting cell proliferation and inducing cell cycle arrest and apoptosis, suggesting that Bmi-1 may serve as a potential and specific novel therapeutic target in MM.

\section{Materials and methods}

Cell culture. HEK-293T cells were cultured in Dulbecco's modified Eagle's medium containing $10 \%$ fetal bovine serum. U266 and RPMI8226 cells were cultured in RPMI-1640 containing $10 \%$ fetal bovine serum, $50 \mathrm{U} / \mathrm{ml}$ penicillin and $50 \mu \mathrm{g} / \mathrm{ml}$ streptomycin.

Lentivirus production and infection. Bmi-1 shRNA was designed and cloned into the pLVTHM lentiviral vector. The shRNA sequence for Bmi-1 was as follows: 5'-GAGATAATAA GCTTGTCTA-3' (26). A shRNA targeting a scrambled sequence (general sequence, 5'-TTCTCCGAACGTGTCACGT-3') (27) served as the negative control. The clone identity was verified by restriction digestion analysis and plasmid DNA sequencing. The pWPXL vectors were transfected into the HEK-293T cells with the packaging plasmid, psPAX2, and the VSV-G envelope plasmid, pMD2.G (a gift from Dr Didier Trono, School of Life Sciences, Ecole Polytechnique Fédérale de Lausanne, Lausanne, Switzerland), using Lipofectamine 2000 (Invitrogen, Carlsbad, CA, USA). The viral particles were harvested $48 \mathrm{~h}$ following transfection. The U266 and RPMI8226 cells $\left(1 \times 10^{5}\right)$ were infected with $1 \times 10^{6}$ recombinant lentivirus-transducing units plus $6 \mu \mathrm{g} / \mathrm{ml}$ of polybrene (Sigma, Natick, MA, USA).

$R N A$ extraction and quantitative reverse transcription polymerase chain reaction ( $R T-q P C R)$. Total RNA was extracted from the U266 and RPMI8226 cells using TRIzol reagent (Invitrogen) and reverse transcribed to generate cDNA. cDNA was synthesized using the PrimeScript RT Reagent kit (Takara, Tokyo, Japan). qPCR analyses were performed using SYBR Premix Ex Taq (Takara). The primers used for the PCR amplification were as follows: Bmi-1 forward, 5'-AAATCA GGGGGTTGAAAAAT CT-3' and reverse, 5'-GCTAACCA
CCAATCTTCCTTTG-3'; p14 forward, 5'-GCTACTGAGGAG CCAGCGTCTA-3' and reverse, 5'-AGCACCACCAGCGTGT CCAG-3'; and $\beta$-actin forward, 5'-GCTACTGAGGAGCCAG CGTCTA-3' and reverse, 5'-AGCACCACCAGCGTGTCCAG-3'.

Western blot analysis. The proteins were separated on a $12 \%$ SDS-PAGE gels and then transferred onto a nitrocellulose membrane (Bio-Rad, Hercules, CA, USA). The membrane was blocked using $5 \%$ non-fat milk and incubated with a mouse anti-Bmi-1, anti-p21 or anti-Bcl-2 monoclonal antibody (mAb) (Cell Signaling Technology, Inc., Beverly, MA, USA) or a mouse anti- $\beta$-actin mAb (Sigma). The proteins were visualized and quantified using ECL reagents (Pierce Biotechnology, Inc., Rockford, IL, USA).

Cell proliferation. Cell proliferation was measured using the cell counting kit-8 (CCK-8) assay kit (Dojindo Corp., Kumamoto, Japan). A total of 4,000 cells were plated into each well of a 96-well plate, wherein $10 \mu \mathrm{l}$ of the CCK-8 solution was added to $90 \mu \mathrm{l}$ of the culture medium. The cells were subsequently incubated for $2 \mathrm{~h}$ at $37^{\circ} \mathrm{C}$, and the optical density was measured at 450 and $650 \mathrm{~nm}$. Three independent experiments were performed.

FACS analysis for determination of cell cycle progression and apoptosis. The U266 and RPMI8226 cells were collected and fixed in ice-cold $70 \%$ ethanol overnight. The fixed cells were washed with phosphate-buffered saline and stained with a freshly prepared solution containing $25 \mu \mathrm{g} / \mathrm{ml}$ propidium iodide (PI; Sigma), $10 \mu \mathrm{g} / \mathrm{ml}$ RNase A, $0.05 \mathrm{mM}$ ethylene diamine and $0.2 \%$ Triton $\mathrm{X}-100$ tetra-acetic acid in phosphatebuffered saline for $30 \mathrm{~min}$ in the absence of light. For each sample, at least 20,000 cells were analyzed using a flow cytometer (Beckman Coulter EPICS Altra Cell Sorter; Beckman Coulter Inc., Miami, FL, USA) and Multicycle AV software for Windows 5.0 (Phoenix Flow Systems, San Diego, CA, USA).

Cells $\left(2 \times 10^{5}\right)$ from the control group and the interference group were collected, stained according to the instructions provided with the Annexin V-PE/7-AAD Apoptosis Detection kit (Sigma), and subjected to flow cytometry (FCM) using a FACSCalibur flow cytometer (BD Biosciences, San Jose, CA, USA) for the detection of apoptosis.

Statistical analysis. The results in this study are presented as the means \pm SEM. The data were analyzed using the Student's t-test (two-tailed) and a value of $\mathrm{p}<0.05$ was considered to indicate a statistically significant difference, unless otherwise specified.

\section{Results}

Establishment of cell lines stably transfected with shRNA targeting Bmi-1 (shBmi-1). To determine the biological role of Bmi-1 in the survival of MM cells, two cell lines stably transfected with shBmi-1 were established. The results from RT-qPCR demonstrated that Bmi-1 mRNA expression in the shBmi-1-transfected cells was significantly lower than that in the control cells ( $\mathrm{p}<0.05$; Fig. 1A and B). Additionally, western blot analysis revealed that the Bmi-1 protein levels were markedly reduced in the shBmi-1-transfected cells (Fig. 1C and D), demonstrating that the constructed lentivirus-mediated RNAi 

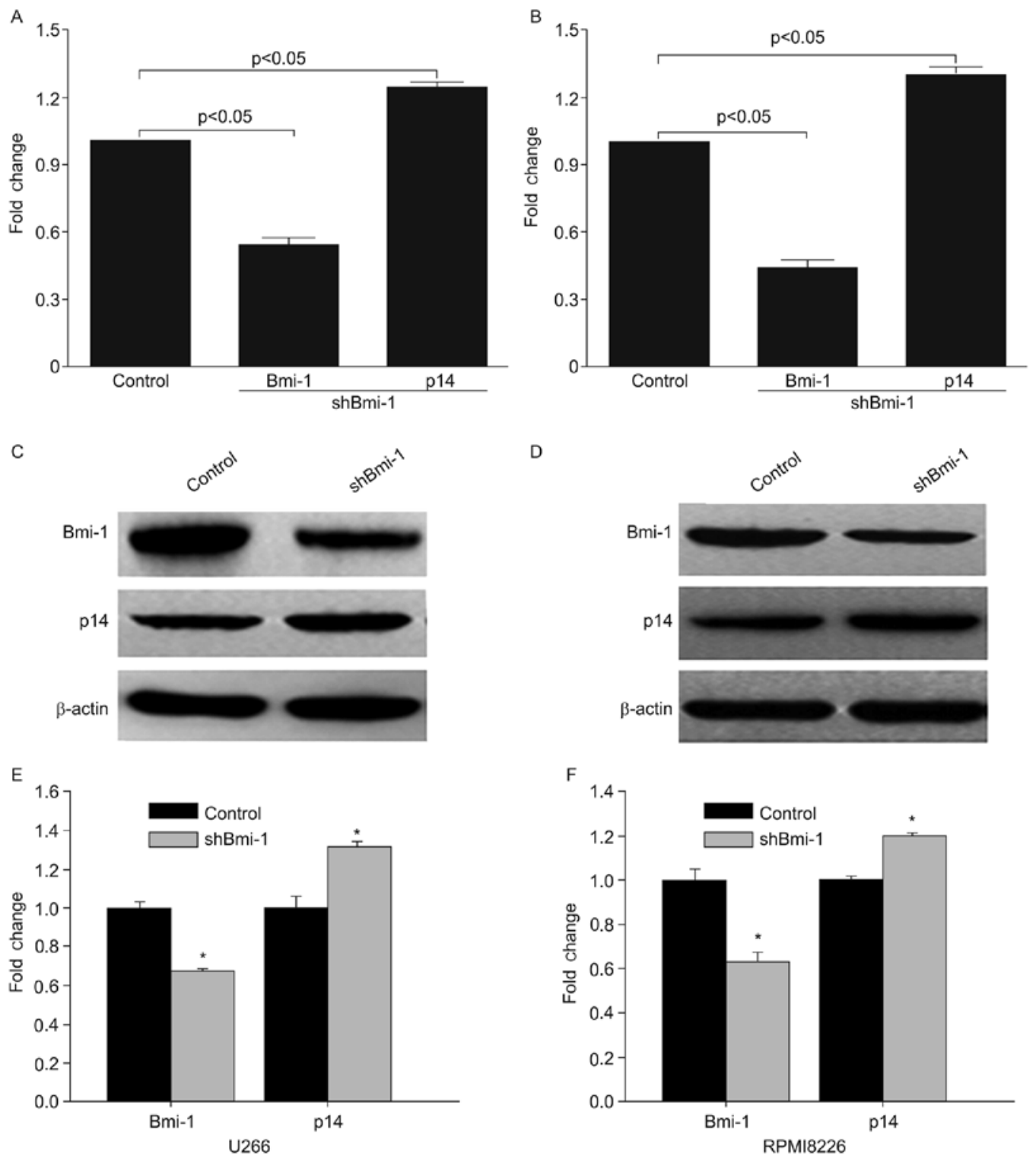

D
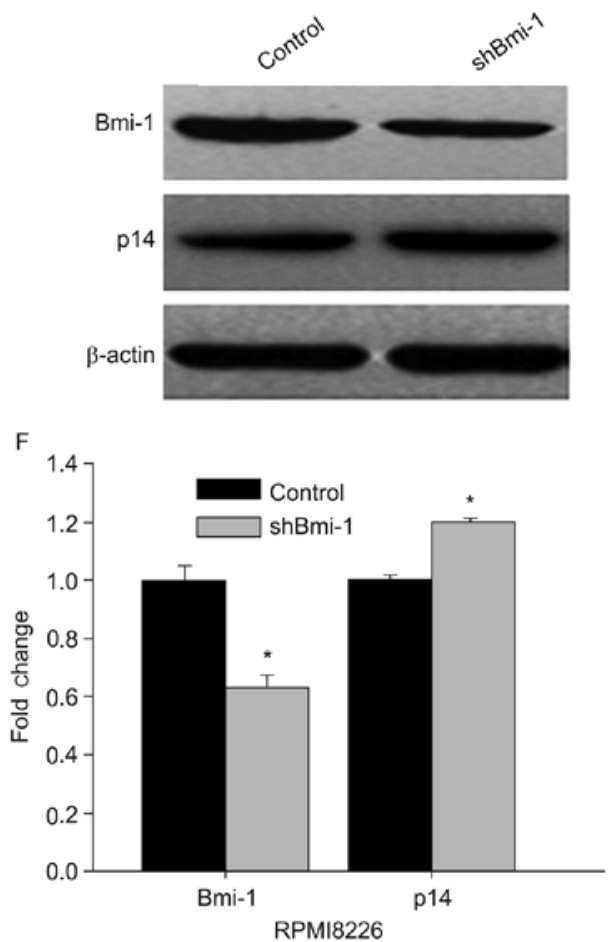

Figure 1. Effect of short hairpin RNA (shRNA) targeting B-cell-specific Moloney murine leukemia virus insertion site-1 (Bmi-1; shBmi-1)) on the mRNA and protein expression of Bmi-1 and p14. (A, C and E) U266 cells; (B, D and F) RPMI8226 cells. (A and B) mRNA expression levels of Bmi-1 and p14 determined by RNA extraction and RT-qPCR following lentivirus transfusion. $\beta$-actin was used for normalization, and the control values were normalized to 1 for comparative analysis. (C and D) Western blot analysis of Bmi-1 and p14 protein levels following lentivirus transfusion. (E and F) Densitometric analysis. $\beta$-actin served as the internal reference. Triplicate experiments showed consistent results. ${ }^{*} \mathrm{p}<0.05$, vs. control.

expression vector expressingBmi-1 (shBmi-1) inhibited the expression of Bmi-1. Bmi-1 has been shown to inhibit the INK4a/ARF locus, which exerts a significant negative effect on p14ARF transcriptional regulation (10). By detecting p14 gene expression, we established that the reduced expression of Bmi-1 resulted in an increased p14 mRNA and protein expression (Fig. 1). These results demonstrate that shRNA technology can be used to effectively inhibit Bmi-1 mRNA and protein expression in myeloma cells, indicating that myeloma cell lines in which the expression of Bmi-1 was effectively silenced by RNAi were created successfully.

Bmi-1 knockdown sensitizes U266 and RPMI8226 cells to bortezomib. To examine the effects of bortezomib on the survival of cells in which Bmi-1 was knocked down , a CCK-8 assay was used to detect the proliferation of U266-shBmi-1, RPMI8226-shBmi-1, U266-control and RPMI8226-control cells. The results indicated that the silencing of the Bmi-1 gene gradually enhanced the inhibition of MM cell proliferation in a time-dependent manner compared to the control (Fig. 2A and B). Subsequently, the stably transfected cell lines were treated with various concentrations $(0,10,20,40$ and $80 \mathrm{nM})$ of bortezomib for $72 \mathrm{~h}$, and a CCK-8 assay was performed in order to detect the proliferative ability of the cells. The results indicated a marked inhibition of cell proliferation in the shBmi-1-transfected cells compared with the control group (Fig. 2C and D). The 50\% inhibitory concentration (IC50) values of bortezomib in the U266-control and U266-shBmi-1 cells were $24.73 \pm 0.375 \mathrm{nM}$ and $18.59 \pm 0.286 \mathrm{nM}(\mathrm{p}<0.05)$, respectively. The IC50 values of bortezomib in the RPMI8226-control and RPMI8226-shBmi-1 

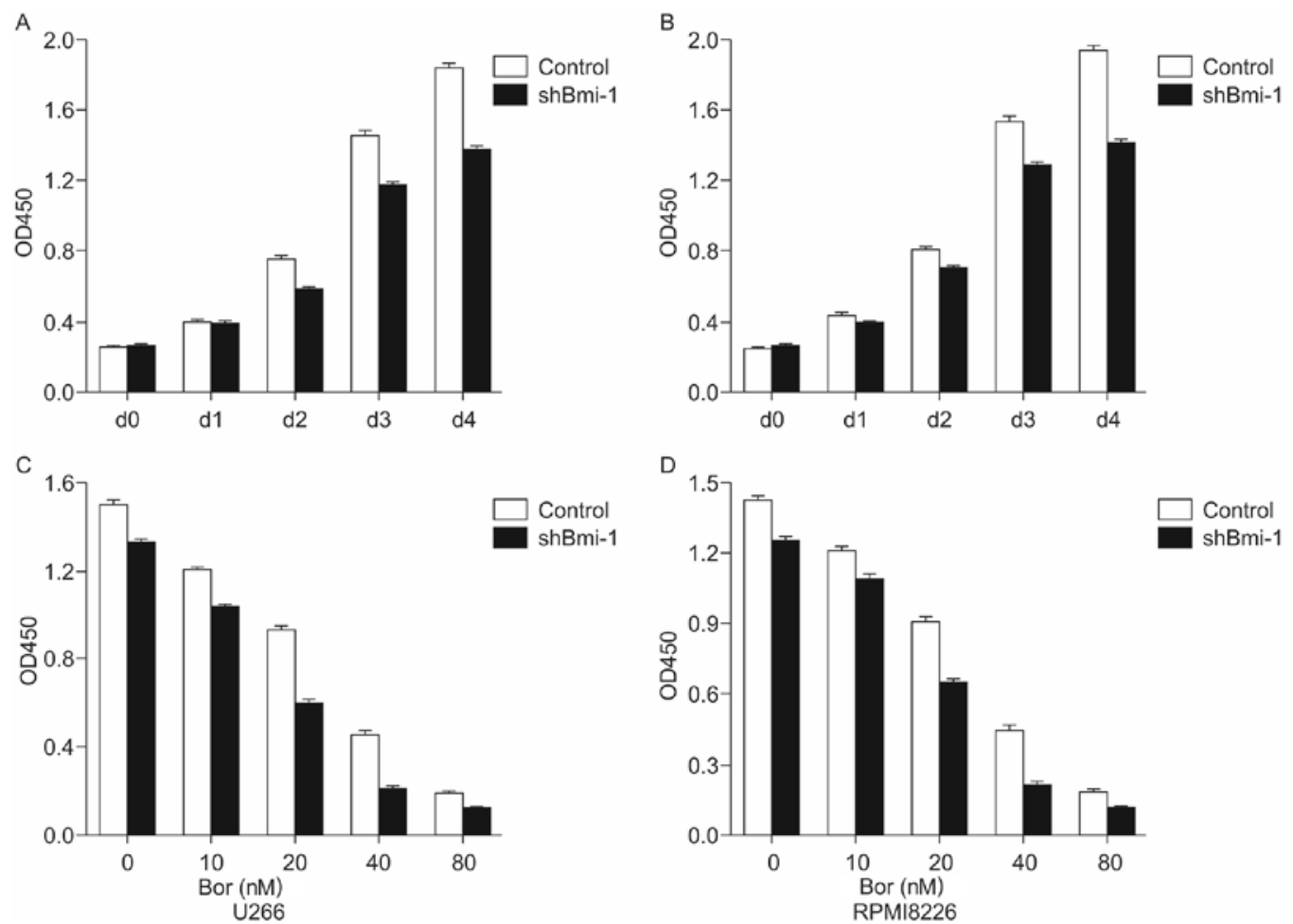

Figure 2. Inhibitory effect of short hairpin RNA (shRNA) targeting B-cell-specific Moloney murine leukemia virus insertion site-1 (Bmi-1; shBmi-1) combined with or without bortezomib treatment on multiple myeloma (MM) cells. (A and B) A CCK8 cell proliferation assay of U266-control, U266-shBmi-1, RPMI8226-control and RPMI8226-shBmi-1 cells was performed for $2 \mathrm{~h}$ after 0, 24, 48, 72 and $96 \mathrm{~h}$. (C and D) U266-control, U266-shBmi-1, RPMI8226control, and RPMI8226-shBmi-1 cells were exposed to bortezomib for $72 \mathrm{~h}$, and a CCK8 assay was used to evaluate cell viability. The data are presented as the means \pm SD of three independent experiments. Bor, bortezomib.

cells were $32.99 \pm 0.458 \mathrm{nM}$ and $21.56 \pm 0.526 \mathrm{nM}(\mathrm{p}<0.05)$, respectively. These results indicated that the knockdown of Bmi-1 sensitized the U266 and RPMI8226 cells to bortezomib.

Bmi-1 silencing induces myeloma cell cycle arrest in the $G_{1}$ phase. FCM was used to detect the cell cycle progresion of the U266 and RPMI8226 cells which were stably transfected with shBmi-1 followed by treatment with or without 20 or $30 \mathrm{nM}$ of bortezomib. The $\mathrm{G}_{1}$ distribution rates of the U266-control and U266-shBmi-1 cells were $39.91 \pm 0.26 \%$ and $45.65 \pm 0.68 \%(p<0.05)$, respectively. The $\mathrm{G}_{1}$ distribution rates of the RPMI8226-control and RPMI8226-shBmi-1 cells were $42.30 \pm 0.47 \%$ and $50.86 \pm 0.38 \%(\mathrm{p}<0.05)$, respectively. Furthermore, in combination with bortezomib treatment for $48 \mathrm{~h}$, the silencing of Bmi-1 induced a significant increase in the number of myeloma cells arrested in the $\mathrm{G}_{1}$ phase $(\mathrm{p}<0.05$; Fig. 3). The $\mathrm{G}_{1}$ distribution rates of the U266-control and $\mathrm{U} 266$ shBmi- 1 cells were $45.35 \pm 0.77 \%$ and $56.81 \pm 0.50 \%(\mathrm{p}<0.05)$, respectively. The $\mathrm{G}_{1}$ distribution rates of the RPMI8226control and RPMI8226-shBmi-1 cells were $49.27 \pm 0.90 \%$ and $56.94 \pm 0.61 \%(\mathrm{p}<0.05)$, respectively. These data demonstrate that the silencing of Bmi-1 enhances the effects of bortezomib on cell cycle arrest.

Bmi-1 silencing enhances the apoptotic effects of bortezomib. Annexin V-PE/7-AAD staining was utilized to evaluate the effects of Bmi-1 knockdown on MM cell apoptosis. The results indicated that the percentage of U266-control and
U266-shBmi-1 apoptotic cells was 9.8 and $12.12 \%(\mathrm{p}<0.05)$, respectively, and the percentage of RPMI8226-control and RPMI8226-shBmi-1 apoptotic cells was 9.6 and $15.69 \%$ $(\mathrm{p}<0.05)$, respectively (Fig. 4). Furthermore, we examined the apoptotic rate of the U266-shBmi-1 and U266-control cells as well as that of the RPMI8226-shBmi-1 and RPMI8226-control cells following treatment with 20 or $30 \mathrm{nM}$ bortezomib for $72 \mathrm{~h}$. The FCM data demonstrated that the apoptotic rate of the U266-shBmi-1 and RPMI8226-shBmi-1 cells was 24.55 and $49.14 \%$, respectively, compared with 20.83 and $22.50 \%$ of the U266-control and RPMI8226-control cells, respectively ( $<<0.05$; Fig. 4). These observations indicated that the silencing of Bmi-1 enhanced the ability of bortezomib to induce MM cell apoptosis.

Bmi-1 silencing combined with bortezomib regulates the expression levels of p21, Bax and Bcl-2. To further explore the mechanisms underlying the enhancement of bortezomib-induced apoptosis in Bmi-1-silenced cells, we examined the expression levels of p21, Bax and Bcl-2 in the U266-shBmi-1 and U266-control cells (treated with or without $20 \mathrm{nM}$ bortezomib) and in the RPMI8226-shBmi-1 and RPMI8226-control cells (treated with or without $30 \mathrm{nM}$ bortezomib). Compared with the control group, the protein expression levels of p21 and Bax in the shBmi-1 cells were significantly increased, while the expression of the anti-apoptotic protein, Bcl-2, was significantly reduced ( $<<0.05$; Fig. 5). Furthermore, when combined with the results obtained at $48 \mathrm{~h}$ 

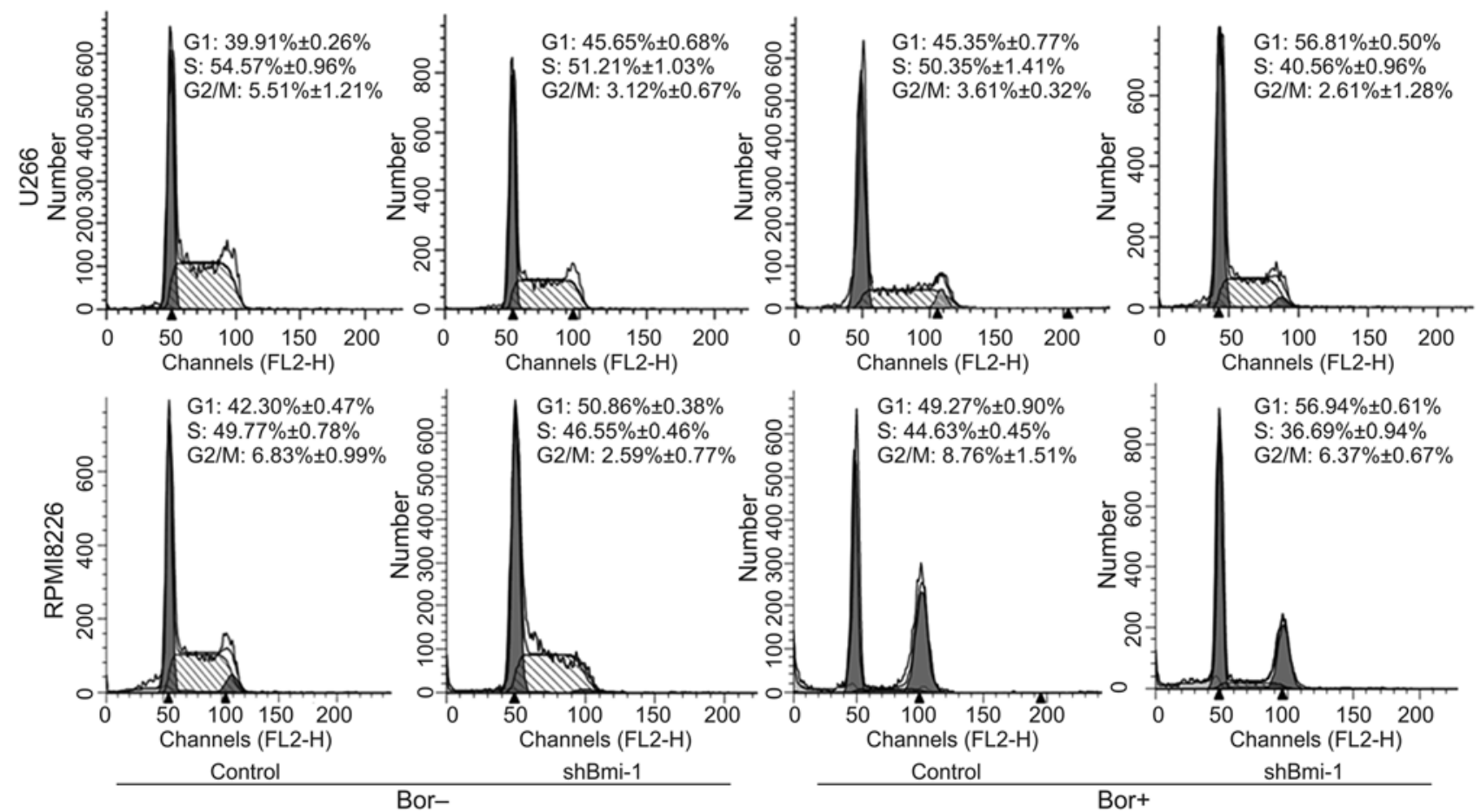

Figure 3. FACS analysis of cell cycle distribution. U266-control, U266-shBmi-1, RPMI8226-control, and RPMI8226-shBmi-1 cells were treated with (Bor+) or without 20 or $30 \mathrm{nM}$ bortezomib (Bor-) for $48 \mathrm{~h}$, and the cells were harvested for propidium iodide (PI) staining followed by flow cytometry (FCM). The percentage of cells displaying $\mathrm{G}_{1}, \mathrm{~S}$, and $\mathrm{G}_{2} / \mathrm{M}$ phase content are provided on each cell cycle figure. The data are presented as the means $\pm \mathrm{SD}$ of three independent experiments. shBmi-1, small interfering RNA targeting B-cell-specific Moloney murine leukemia virus insertion site-1.
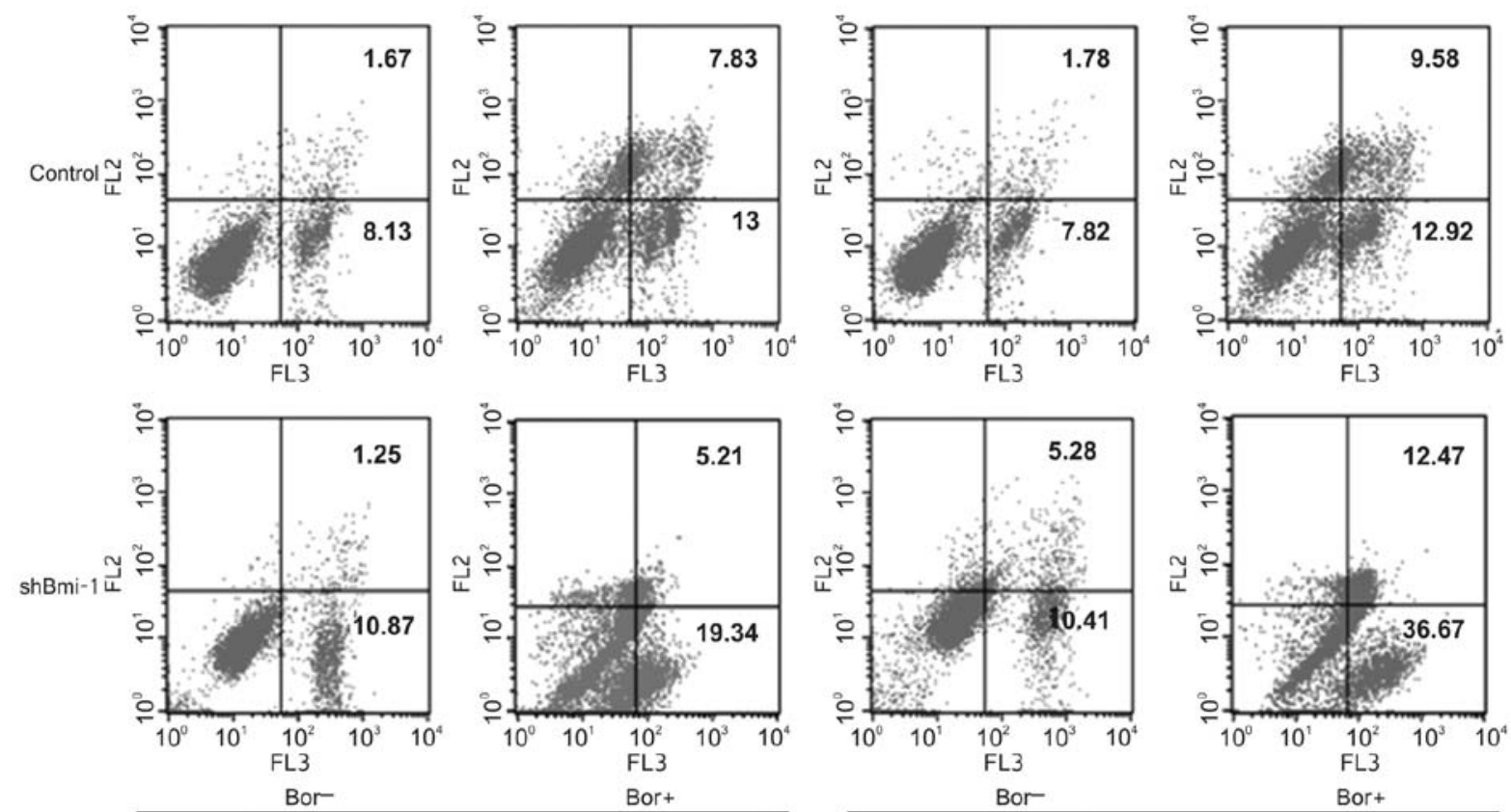

U266

RPMI8226

Figure 4. Silencing of B-cell-specific Moloney murine leukemia virus insertion site 1 (Bmi-1) combined with or without bortezomib treatment affects cell apoptosis of multiple myeloma (MM) cells. U266-control, U266-shBmi-1, RPMI8226-control and RPMI8226-shBmi-1 cells were treated with (Bor+) or without (Bor-) 20 or $30 \mathrm{nM}$ bortezomib for $48 \mathrm{~h}$, and the cells were harvested for Annexin V-PE/7-AAD staining followed by flow cytometry (FCM). The values shown in the lower-right quadrant and upper-right quadrant represent the percentage of early and late-apoptotic cells, respectively.

of bortezomib treatment, the difference was even more evident. These results suggest that the robust increase in p21 and Bax expression and the prominent decrease in Bcl-2 expression enhanced the sensitivity of MM cells to bortezomib. 

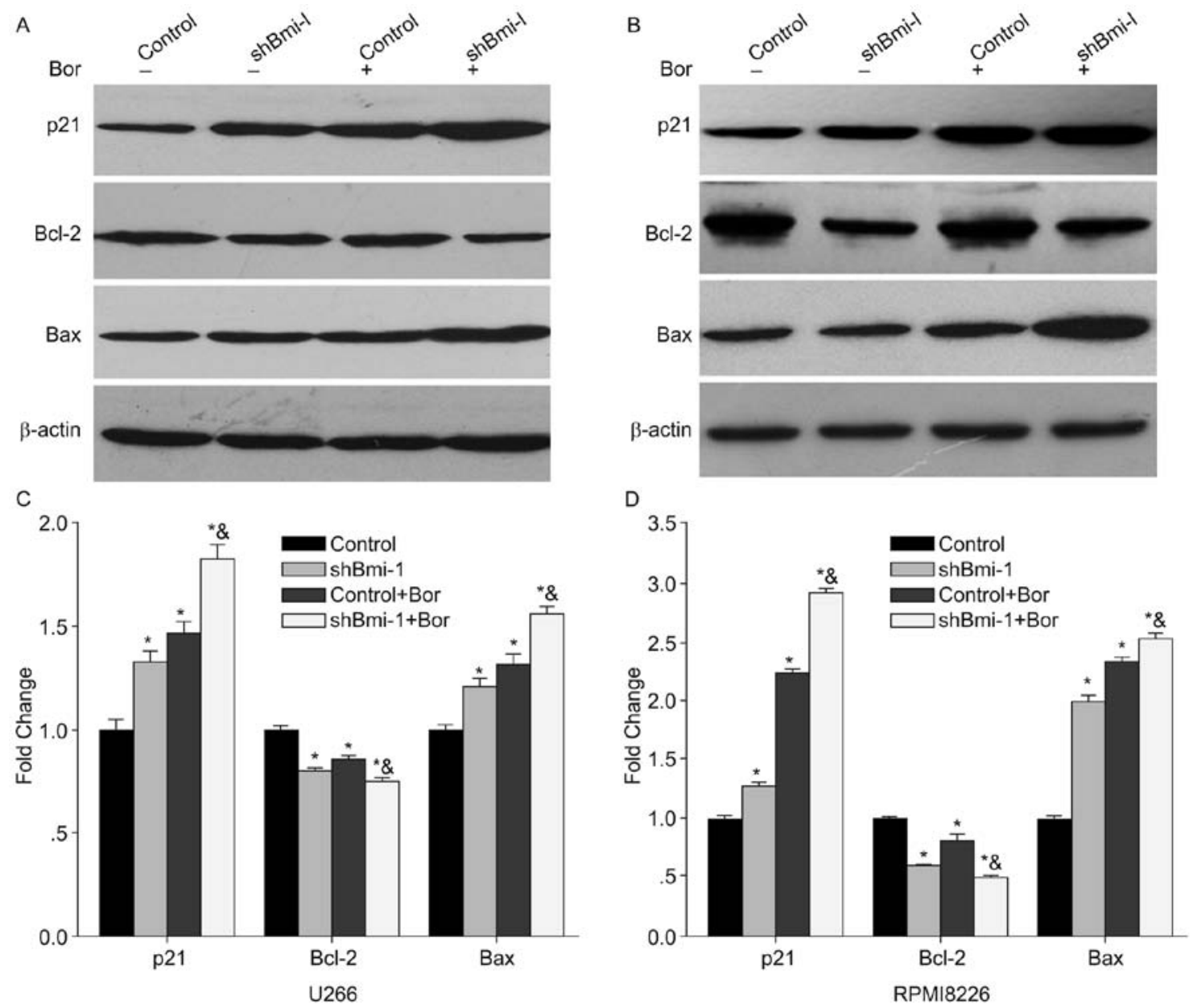

Figure 5. Effect of short hairpin RNA (shRNA) targeting B-cell-specific Moloney murine leukemia virus insertion site 1 (Bmi-1; shBmi-1) combined with bortezomib treatment on the protein expression of p21, Bcl-2 and Bax in multiple myeloma (MM) cells. (A and C) U266 cells; (B and D) RPMI8226 cells. (A and B) U266-control, U266-shBmi-1, RPMI8226-control and RPMI8226-shBmi-1 cells were incubated for $48 \mathrm{~h}$ in the presence (+) or absence (-) of 20 or $30 \mathrm{nM}$ bortezomib (Bor). Total protein was electrophoresed and separated on a 12\% SDS-polyacrylamide gels. The protein expression levels of p21, Bcl-2 and Bax were determined by western blot analysis. (C and D) Densitometric analysis. Triplicate experiments demonstrated consistent results. "p $<0.05$, vs. control. ${ }^{\&} \mathrm{p}<0.05$, compared to control + Bor.

\section{Discussion}

MM is a malignant mature $\mathrm{B}$ cell neoplasm characterized by the clonal proliferation of plasma cells in BM, which is characterized by a profound genomic instability involving both numerical and structural chromosomal aberrations of potential prognostic relevance (28). As an incurable cancer, MM can occur de novo or proceed from a monoclonal gammopathy of undetermined significance (MGUS; 1\% per year) (4). Multilevel molecular changes, such as the activation of oncogenes, inactivation of tumor suppressor genes, and alteration of the BM microenvironment, are involved in the pathogenesis and progression of MM. Due to comprehensive advances in the understanding of the molecular pathogenesis of MM, some therapeutic medicines, such as protease inhibitors, lenalidomide and farnesyl transferase inhibitors, that target signaling pathways or the BM microenvironment have been introduced to clinical practice, and have significantly improved the survival and prognosis of patients with MM (2). Among the therapeutic drugs, bortezomib, which is a proteasome inhibitor, has been shown to specifically inhibit the $26 \mathrm{~S}$ proteasome subunit and I- $\kappa \mathrm{B}$ degradation, prevent NF- $\mathrm{B}$ activation, and induce apoptosis in MM. It has also been shown to have marked clinical activity in the relapsed or refractory MM setting (5). In the present study, we also demonstrated that bortezomib induced a dose- and time-dependent increase in cell toxicity and a decrease in the viability of MM cells. Despite a promising clinical activity, some patients with MM have failed to respond to bortezomib therapy. Moreover, only 25-35\% of patients respond to bortezomib treatment in the relapsed and refractory setting $(3,4)$. Therefore, to improve the outcome of this incurable disease, further research is required to overcome resistance to bortezomib in MM. Studies have demonstrated that the administration of bortezomib in combination with other chemical medications can sensitize cancer cells to bortezomib or even reverse resistance $(29,30)$. Furthermore, it has been reported that the RNAi-mediated silencing of the oncogenes, Mcl-1 and MAGE-C1/CT7, can also sensitize MM cells to bortezomib, suggesting that RNAi may be a potential method for enhancing the sensitivity of MM cells to bortezomib.

Bmi-1, the first polycomb-group gene to be identified, was originally isolated as an oncogenic partner of c-Myc in the initiation of lymphoma in murine models. There is accumulating evidence confirming that Bmi-1 plays a crucial role in diverse 
biological and pathological processes, such as axial patterning, hematopoiesis, the regulation of proliferation, senescence and the maintenance of cancer stem cell self-renewal. Consistent with its role in inhibiting the p16ink4a locus, Bmi-1 is upregulated extensively in a variety of malignancies, including MM, and can regulate cell proliferation and carcinogenesis. Previous studies have also suggested that Bmi-1 is associated with the protection of tumor cells from apoptosis $(31,32)$. Moreover, the increased expression of Bmi-1 in certain tumors correlates with a poor prognosis $(20,32,33)$. Additionally, increasing evidence indicates that the RNAi-mediated depletion of Bmi-1 can sensitize tumor cells to chemotherapy or radiotherapy. In an attempt to enhance sensitivity to bortezomib, these observations prompted us to investigate the possibility of combining Bmi-1 silencing and bortezomib treatment as a clinical therapeutic strategy for MM.

In order to evaluate the possibility of developing Bmi-1 into a novel therapeutic agent for the treatment of MM, two MM cell lines (U266 and RPMI8226), which overexpress Bmi-1, were employed in the present study. A lentiviral interference vector expressing shRNA was introduced into the U266 and RPMI8226 cells, effectively silencing Bmi- 1 . We established that the proliferation of U266 and RPMI8226 cells was markedly inhibited after the silencing of Bmi-1, and the depletion of Bmi-1 resulted in an enhanced sensitivity of these cells to bortezomib. FACS observations revealed that silencing Bmi-1 expression in combination with bortezomib treatment prompted cell cycle arrest in the $\mathrm{G}_{1}-\mathrm{S}$ phase and enhanced bortezomib-induced apoptosis. These results indicate that the silencing of Bmi-1 sensitizes MM cells to bortezomib by inhibiting cell proliferation and inducing cell cycle arrest and apoptosis.

Bortezomib induces apoptosis by stabilizing p53 and activating p53 downstream target genes, such as p21. However, bortezomib can induce apoptosis in cells that do not contain wild-type p53 and the effect of bortezomib on p21 may actually limit its direct cytotoxic activities (34). Furthermore, bortezomib may selectively induce apoptosis in SV40-transformed, but not in normal, fibroblasts, although the accumulation of $\mathrm{p} 21$ occurs in both cell types (35). Therefore, the accumulation of p21 may be involved in, but is not absolutely required for, the induction of apoptosis. However, p21, a cyclin-dependent kinase inhibitor, can induce growth arrest by inhibiting cyclin-dependent kinases that drive cell cycle progression, sensitizing cancer cells to chemotherapy (36). The Bcl-2 family is comprised of structurally related proteins that can either inhibit (i.e., Bcl-2) or promote (i.e., Bax) cell death (37). The ratio of Bcl-2 to Bax is a key regulator for determining apoptosis following damage to DNA. Proteasome inhibitors can induce apoptosis by inducing an accumulation of pro-apoptotic Bcl-2 family members. In addition, evidence indicates that bortezomib overcomes the protective effects of $\mathrm{Bcl}-2$ overexpression by inhibiting the degradation of $\mathrm{Bax}(38,39)$. However, alterations in the balance between Bcl-2 and Bax may be involved in resistance to bortezomib (40). Therefore, in this study,. we focused on the expression levels of $\mathrm{p} 21, \mathrm{Bcl}-2$ and Bax. The results indicated that the knockdown Bmi-1 resulted in the upregulation of $\mathrm{p} 21$, the downregulation of $\mathrm{Bcl}-2$ and the accumulation of Bax in the cells treated with bortezomib. These findings suggest that p21 is involved in the Bmi-1-driven cell proliferation inhibition and
$\mathrm{G}_{1}$ phase arrest in MM cells. Furthermore, the results indicated that Bmi-1 downregulated the Bcl-2/Bax ratio and enhanced bortezomib-induced apoptosis.

In conclusion, to the best of our knowledge, this is the first report of the the anticancer potential of bortezomib treatment combined with the depletion of Bmi-1 in MM. The present study demonstrates that the knockdown of Bmi-1 sensitizes MM cells to bortezomib treatment and that the silencing of Bmi-1 enhances bortezomib-induced apoptosis. Furthermore, we demonstrate that the knockdown of Bmi-1 upregulates the expression of $\mathrm{p} 21$ and $\mathrm{Bcl}-2$ and decreases the expression of Bax. The alteration in the $\mathrm{Bcl}-2 / \mathrm{Bax}$ ratio prompted bortezomib-induced apoptosis in Bmi-1-silenced MM cells. Taken together, our data suggest that bortezomib treatment combined with Bmi-1 knockdown may be a potential therapeutic strategy for MM.

\section{Acknowledgements}

We are most grateful to Dr Didier Trono for offering the pWPXL, psPAX2 and pMD2.G lentivirus plasmids as gifts. The present study was supported by grants from the National Natural Science Foundation of China (no. 81201872), the Natural Science Foundation of Fujian Province (no. 2013J01308), the Foundation of Fujian Key Laboratory of Hematology (no. 2009J1004), and the Fujian Provincial Health Bureau Youth Research Projects (no. 2010-1-11).

\section{References}

1. Kyle RA and Rajkumar SV: Multiple myeloma. N Engl J Med 351: 1860-1873, 2004.

2. Mitsiades CS, Mitsiades N, Munshi NC and Anderson KC: Focus on multiple myeloma. Cancer Cell 6: 439-444, 2004.

3. Bergsagel PL and Kuehl WM: Molecular pathogenesis and a consequent classification of multiple myeloma. J Clin Oncol 23: 6333-6338, 2005.

4. Hideshima T, Mitsiades C, Tonon G, Richardson PG and Anderson KC: Understanding multiple myeloma pathogenesis in the bone marrow to identify new therapeutic targets. Nat Rev Cancer 7: 585-598, 2007.

5. Richardson PG, Barlogie B, Berenson J, et al: A phase 2 study of bortezomib in relapsed, refractory myeloma. N Engl J Med 348: 2609-2617, 2003.

6. Kumar SK, Rajkumar SV, Dispenzieri A, et al: Improved survival in multiple myeloma and the impact of novel therapies. Blood 111: 2516-2520, 2008.

7. Cheriyath V, Jacobs BS and Hussein MA: Proteasome inhibitors in the clinical setting: benefits and strategies to overcome multiple myeloma resistance to proteasome inhibitors. Drugs RD 8: 1-12, 2007.

8. de Carvalho F, Costa ET, Camargo AA, et al: Targeting MAGE-C1/CT7 expression increases cell sensitivity to the proteasome inhibitor bortezomib in multiple myeloma cell lines. PLoS One 6: e27707, 2011.

9. Podar K, Gouill SL, Zhang J, et al: A pivotal role for Mcl-1 in Bortezomib-induced apoptosis. Oncogene 27: 721-731, 2008.

10. Jacobs JJ, Kieboom K, Marino S, DePinho RA and van Lohuizen M: The oncogene and Polycomb-group gene bmi-1 regulates cell proliferation and senescence through the ink4a locus. Nature 397: 164-168, 1999.

11. Jacobs JJ, Scheijen B, Voncken JW, Kieboom K, Berns A and van Lohuizen M: Bmi-1 collaborates with c-Myc in tumorigenesis by inhibiting c-Myc-induced apoptosis via INK4a/ARF Genes Dev 13: 2678-2690, 1999.

12. Leung C, Lingbeek M, Shakhova O, et al: Bmil is essential for cerebellar development and is overexpressed in human medulloblastomas. Nature 428: 337-341, 2004.

13. Park IK, Qian D, Kiel M, et al: Bmi-1 is required for maintenance of adult self-renewing haematopoietic stem cells. Nature 423: 302-305, 2003. 
14. Molofsky AV, He S, Bydon M, Morrison SJ and Pardal R: Bmi-1 promotes neural stem cell self-renewal and neural development but not mouse growth and survival by repressing the p16Ink4a and p19Arf senescence pathways. Genes Dev 19: 1432-1437, 2005.

15. Yang J, Chai L, Liu F, et al: Bmi-1 is a target gene for SALL4 in hematopoietic and leukemic cells. Proc Natl Acad Sci USA 104 10494-10499, 2007.

16. Vonlanthen S, Heighway J, Altermatt HJ, et al: The bmi-1 oncoprotein is differentially expressed in non-small cell lung cancer and correlates with INK4A-ARF locus expression. Br J Cancer 84: 1372-1376, 2001.

17. Larmonie NS, Dik WA, Beverloo HB, van Wering ER, van Dongen JJ and Langerak AW: BMI1 as oncogenic candidate in a novel TCRB-associated chromosomal aberration in a patient with TCRgammadelta+ T-cell acute lymphoblastic leukemia. Leukemia 22: 1266-1267, 2008.

18. Choi YJ, Choi YL, Cho EY, et al: Expression of Bmi-1 protein in tumor tissues is associated with favorable prognosis in breast cancer patients. Breast Cancer Res Treat 113: 83-93, 2009.

19. Kim JH, Yoon SY, Kim CN, et al: The Bmi-1 oncoprotein is overexpressed in human colorectal cancer and correlates with the reduced p16INK4a/p14ARF proteins. Cancer Letters 203 217-224, 2004

20. Song W, Tao K, Li H, et al: Bmi-1 is related to proliferation, survival and poor prognosis in pancreatic cancer. Cancer Sci 101: $1754-1760,2010$

21. van Leenders GJ, Dukers D, Hessels D, et al: Polycomb-group oncogenes EZH2, BMI1, and RING1 are overexpressed in prostate cancer with adverse pathologic and clinical features. Eur Urol 52: 455-463, 2007

22. Jagani Z, Wiederschain D, Loo A, et al: The Polycomb group protein Bmi-1 is essential for the growth of multiple myeloma cells. Cancer Res 70: 5528-5538, 2010.

23. Crea F, Duhagon Serrat MA, Hurt EM, Thomas SB, Danesi R and Farrar WL: BMI1 silencing enhances docetaxel activity and impairs antioxidant response in prostate cancer. Int J Cancer 128: 1946-1954, 2011

24. Wang E, Bhattacharyya S, Szaboles A, et al: Enhancing chemotherapy response with Bmi-1 silencing in ovarian cancer. PLoS One 6: e17918

25. Wu J, Hu D, Yang G, et al: Down-regulation of BMI-1 cooperates with artemisinin on growth inhibition of nasopharyngeal carcinoma cells. J Cell Biochem 112: 1938-1948, 2011.

26. Fasano CA, Dimos JT, Ivanova NB, Lowry N, Lemischka IR and Temple S: shRNA knockdown of Bmi-1 reveals a critical role for p21-Rb pathway in NSC self-renewal during development. Cell Stem Cell 1: 87-99, 2007.

27. Huang BB, Gao QM, Liang W, Xiu B, Zhang WJ and Liang AB: Down-regulation of SENP1 expression increases apoptosis of Burkitt lymphoma cells. Asian Pac J Cancer Prev 13: 2045-2049, 2012.
28. Fonseca R, Barlogie B, Bataille R, et al: Genetics and cytogenetics of multiple myeloma: a workshop report. Cancer Res 64 : 1546-1558, 2004

29. Briani C, Berno T, Campagnolo $\mathrm{M}$ and Zambello $\mathrm{R}$ Lenalidomide for bortezomib-resistant multiple myeloma. Nat Rev Clin Oncol 7. doi: 10.1038/nrclinonc.2010.31-c1, 2010.

30. Hideshima T, Bradner JE, Wong J, et al: Small-molecule inhibition of proteasome and aggresome function induces synergistic antitumor activity in multiple myeloma. Proc Natl Acad Sci USA 102: 8567-8572, 2005.

31. Liu L, Andrews LG and Tollefsbol TO: Loss of the human polycomb group protein BMI1 promotes cancer-specific cell death. Oncogene 25: 4370-4375, 2006.

32. Wang H, Pan K, Zhang HK, et al: Increased polycomb-group oncogene Bmi-1 expression correlates with poor prognosis in hepatocellular carcinoma. J Cancer Res and Clin Oncol 134: 535-541, 2008.

33. Mihara K, Chowdhury M, Nakaju N, et al: Bmi-1 is useful as a novel molecular marker for predicting progression of myelodysplastic syndrome and patient prognosis. Blood 107: 305-308, 2006.

34. Yu J, Tiwari S, Steiner P and Zhang L: Differential apoptotic response to the proteasome inhibitor Bortezomib [VELCADE, PS-341] in Bax-deficient and p21-deficient colon cancer cells. Cancer Biol Ther 2: 694-699, 2003.

35. An B, Goldfarb RH, Siman R and Dou QP: Novel dipeptidyl proteasome inhibitors overcome $\mathrm{Bcl}-2$ protective function and selectively accumulate the cyclin-dependent kinase inhibitor p27 and induce apoptosis in transformed, but not normal, human fibroblasts. Cell Death Differ 5: 1062-1075, 1998

36. Lazzarini R, Moretti S, Orecchia S, Betta PG, Procopio A and Catalano A: Enhanced antitumor therapy by inhibition of p21waf1 in human malignant mesothelioma. Clin Cancer Res 14: 5099-5107, 2008.

37. Gross A, McDonnell JM and Korsmeyer SJ: BCL-2 family members and the mitochondria in apoptosis. Genes Dev 13: 1899-1911, 1999.

38. Herrmann JL, Briones F Jr, Brisbay S, Logothetis CJ and McDonnell TJ: Prostate carcinoma cell death resulting from inhibition of proteasome activity is independent of functional Bcl-2 and p53. Oncogene 17: 2889-2899, 1998.

39. Tan TT, Degenhardt K, Nelson DA, et al: Key roles of BIM-driven apoptosis in epithelial tumors and rational chemotherapy. Cancer Cell 7: 227-238, 2005.

40. McConkey DJ and Zhu K: Mechanisms of proteasome inhibitor action and resistance in cancer. Drug Resist Updat 11: 164-179, 2008. 